\title{
Management of patients with intramural hematoma involving the ascending aorta
}

\author{
Yoshimasa Moizumi, MDa \\ Tsunehiro Komatsu, MDa \\ Naotaka Motoyoshi, MDa \\ Koichi Tabayashi, MD
}

See related editorial on page 894.
Objective: Although intramural hematoma of the aorta is considered a unique pathologic entity, the management of intramural hematoma involving the ascending aorta (type A) has not been well established. The purpose of this study was to establish the optimal mode of management of type A intramural hematoma.

Methods: We treated patients with type A intramural hematoma as follows. Early operation was carried out only for patients with cardiac tamponade, impending rupture, or rupture. Other patients were treated medically, but patients with progression of intramural hematoma during medical follow-up had their treatment converted to surgery. From February 1992 to March 2001, a total of 33 patients with type A intramural hematoma were treated as described here. Patients were divided according to initial treatment into an early surgery group $(n=9)$ and a medical treatment group $(\mathrm{n}=24)$. Clinical profiles and in-hospital and long-term survival rates were compared between the groups.

Results: Compared with the early surgery group, the medical treatment group was younger $(64.2 \pm 7.0$ years vs $71.7 \pm 8.5$ years, $P=.0319)$ and had a greater number of involved segments $(3.6 \pm 0.6$ vs $3.0 \pm 0.9, P=.0395)$. Eight patients in the medical treatment group were switched to surgery during follow-up because of progression of intramural hematoma. In-hospital mortality rates in the early surgery and medical groups were $11 \%$ and $5 \%(P=.477)$, respectively. Cumulative $1-$ and 2 -year survivals were $89 \%$ and $89 \%$, respectively, in the early surgery group, and $92 \%$ and $81 \%$, respectively, in the medical group $(P=.49)$.

Conclusion: We concluded that about $70 \%$ of type A intramural hematomas could be managed expectantly, and more than $50 \%$ could be treated medically alone.

From the Division of Cardiovascular Surgery, Sendai City Medical Center, ${ }^{a}$ and the Department of Cardiovascular Surgery, Graduate School of Medicine, Tohoku University, ${ }^{\mathrm{b}}$ Sendai, Japan.

Received for publication Jan 30, 2002; revisions requested March 5, 2002; revisions received March 20, 2002; accepted for publication March 29, 2002.

Address for reprints: Yoshimasa Moizumi, $\mathrm{MD}$, the Division of Cardiovascular Surgery, Sendai City Medical Center, 5-22-1 Turugaya, Miyaginoku, Sendai, Miyagi, 983-0824, Japan (E-mail: singe@ openhp. or.jp).

J Thorac Cardiovasc Surg 2002;124:918-24

Copyright $(\odot 2002$ by The American Association for Thoracic Surgery

0022-5223/2002 $\$ 35.00+0 \quad \mathbf{1 2 / 1 / 1 2 5 6 3 7}$

doi:10.1067/mtc.2002.125637

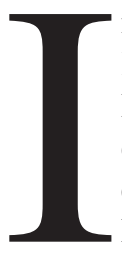

ntramural hematoma of the aorta (IMH) was first described in 1920 by Krukenberg and colleagues ${ }^{1}$ as "dissection without intimal tear." Currently IMH, the clinical presentations of which are similar to those of aortic dissection, is considered a unique pathologic entity..$^{2-8}$ IMH is classified as either involving (type A) or not involving (type B) the ascending aorta. Patients with type B IMH, like those with type B aortic dissection, are usually treated medically. ${ }^{7}$ However, management of patients with type A IMH is controversial. Some reports have recommended early surgery for patients with type A IMH because of their poor prognosis with medical treatment. ${ }^{3-7}$ On the other hand, cases of regression of type A IMH without surgical repair have also been reported. ${ }^{8-10}$ Because of the pathophysiologic process involved in IMH, absence of flow and pressure communication with the aortic lumen may produce good results. ${ }^{20}$ Since 1992, we have treated patients with type A IMH as follows. Early operation is carried out only for patients with cardiac tamponade, impending rupture, or rupture. Patients without tamponade or rupture are treated medically; however, patients with recurrent pain, progression to overt type A dissection, or progressive 
aortic dilatation during medical follow-up have their treatment converted to surgery. The purpose of this study was to evaluate whether our management scheme was reasonable and to attempt to establish the optimal mode of management for type A IMH.

\section{Patients and Methods}

From February 1992 to March 2001, a total of 35 patients were referred to our hospital with the diagnosis of type A IMH. The diagnostic criteria for type A IMH were as follows: (1) symptoms such as severe chest or back pain, such as in acute aortic dissection, (2) initial computed tomographic (CT) examination performed within 24 hours from the onset of symptoms showing the characteristic findings described by Yamada and associates ${ }^{12}$ (displaced intimal calcification, increased external aortic diameter, a crescent high-attenuation area along the aortic wall on plain CT scan, a continuous spiral course of nonopacified soft tissue in the aortic wall on contrast enhanced CT scan, and no evidence of aortic intimal ulceration, disruption, or flap), and (3) involvement of the ascending aorta. The diagnosis of IMH was confirmed by operative findings showing the presence of thrombus or clot in the wall of the ascending aorta and absence of intimal tear from the ascending aorta to distal aortic arch or by serial CT findings showing a morphologic change in aortic wall thickness.

\section{Management of Type A IMH}

Initial management of patients with type A IMH was based on hemodynamic stability and clinical symptoms. Patients with hemodynamic instability or persistent pain (with cardiac tamponade, impending rupture, or rupture) underwent early surgery on an emergency basis. Other patients were treated medically with intensive antihypertensive therapy in the intensive care unit for a few days. Blood pressure in these patients was monitored with an arterial line, and calcium-channel antagonists, nitrates, or $\beta$-adrenergic receptor blockers were administered intravenously to maintain systolic blood pressure between 100 and $130 \mathrm{~mm} \mathrm{Hg}$. Patients were then transferred to the high-care unit, where they rested in bed for 2 weeks. Angiotensin-converting enzyme inhibitors, calcium-channel antagonists, and $\alpha$ - or $\beta$-adrenergic receptor blockers were administered orally to maintain blood pressure between 100 and $140 \mathrm{~mm} \mathrm{Hg}$. These patients remained in the hospital another 2 weeks and were followed up as outpatients as they resumed their previous lifestyle. During the medical treatment, patients with recurrent pain, progression to overt type A dissection, or progressive aortic dilatation of more than $60 \mathrm{~mm}$ had their therapy converted to surgery. Two patients were excluded from surgery, an 88-year-old woman with cardiac tamponade and a 75-year-old man in whom IMH had progressed to overt type A dissection. Both were excluded from this study because they did not conform to our protocol.

\section{Computed Tomographic Examinations}

CT images were obtained at 3- to $10-\mathrm{mm}$ intervals from the aortic arch to the aortic bifurcation, with and without contrast medium. Initial CT scans were performed within 24 hours from the onset of episode in all cases. Serial CT examinations were performed at 2 and 4 weeks after admission and at 3, 6, and 12 months after

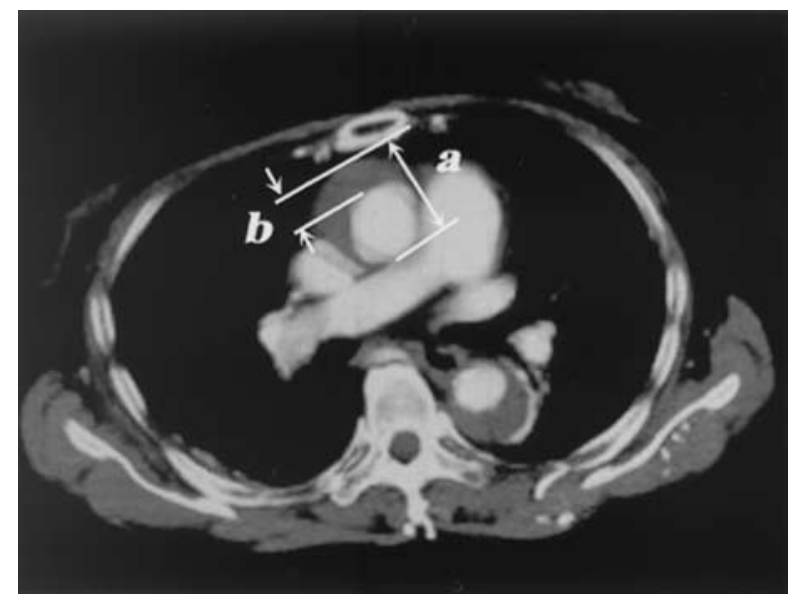

Figure 1. Measurement of aortic dimensions. Maximum external diameter is indicated by $a$ and wall thickness from intima to adventitia is indicated by $b$.

discharge for medically treated patients. The maximum external diameter and wall thickness of the ascending aorta were measured at the level of the right pulmonary artery in the enhanced CT images (Figure 1). The extent of IMH was expressed in numbers of involved segments: involvement of the ascending aorta alone was 1 , that of the ascending aorta and the arch was 2 , that from the ascending to the descending aorta was 3 , and that from the ascending to the abdominal aorta was 4 .

In all but 1 case, follow-up was performed at least once a year by direct or telephone contact or by communication with the primary physician. The follow-up period ranged from 3 months to 114 months (mean $32.5 \pm 30.0$ months).

Of the original 35 patients, 33 were included in this study. On the basis of the initial treatment (within 24 hours after admission), patients were divided into two groups, an early surgery group and a medical treatment group. Clinical profiles and in-hospital and long-term survivals were compared between the groups. For patients in the medical treatment group, clinical characteristics and CT images were analyzed to identify predictors of change from medical treatment to surgery.

\section{Statistical Analysis}

A software package (StatView 5.0 for Macintosh; SAS Institute, Inc, Cary, NC) was used for statistical analysis. Continuous data are presented as mean $\pm \mathrm{SD}$, and categoric data are given as patient numbers and percentages. Continuous variables were evaluated with the Mann-Whitney $U$ test. Discrete variables were treated by means of contingency table and analyzed with the Fisher exact test. The 1- and 2-year survivals were estimated by the Kaplan-Meier method and compared with log-rank tests. The Cox proportional hazards model was used to identify the predictors of change from medical treatment to surgery throughout the entire follow-up period with the use of univariate and forward stepwise Cox regression analysis (entry and removal thresholds of .05 and .1 , respectively). 
TABLE 1. Comparisons of the clinical characteristics and CT findings between the early surgery group and the medical treatment group

\begin{tabular}{lccc}
\hline & $\begin{array}{c}\text { Early surgery } \\
(\mathbf{n}=\mathbf{9})\end{array}$ & $\begin{array}{c}\text { Medical } \\
\text { treatment } \\
(\mathbf{n}=\mathbf{2 4 )}\end{array}$ & $\begin{array}{c}\boldsymbol{P} \\
\text { value }\end{array}$ \\
\hline Age (y) & $71.7 \pm 8.5$ & $64.2 \pm 7.0$ & .032 \\
Sex ratio (male/female) & $5: 4$ & $14: 10$ & $>.999$ \\
Systolic blood pressure & & & \\
$\quad$ on admission & $1(11 \%)$ & $11(46 \%)$ & .107 \\
Over 160 mm Hg & $5(56 \%)$ & $0(0 \%)$ & .001 \\
Below 90 mm Hg & & & \\
Risk factors & $8(89 \%)$ & $22(92 \%)$ & $>.999$ \\
Hypertension & $0(0 \%)$ & $1(4 \%)$ & $>.999$ \\
Diabetes mellitus & $0(0 \%)$ & $2(8 \%)$ & $>.999$ \\
Hyperlipidemia & & & \\
History/associated & $1(11 \%)$ & $6(25 \%)$ & .642 \\
$\quad$ disorders & & & \\
Cerebrovascular & $1(11 \%)$ & $6(25 \%)$ & .642 \\
disease & $1(11 \%)$ & $1(4 \%)$ & .477 \\
Cardiac disease & $1(11 \%)$ & $6(25 \%)$ & .642 \\
Lung disease & $1(11 \%)$ & $4(17 \%)$ & $>.999$ \\
Liver disease & $0(0 \%)$ & $3(13 \%)$ & .545 \\
Renal dysfunction & $0(0 \%)$ & $4(17 \%)$ & .555 \\
Previous IMH & & & \\
Abdominal aortic & & & \\
$\quad$ aneurysm & & & \\
CT examinations on & & & \\
$\quad$ admission & & & \\
Extent of IMH & $3.0 \pm 0.9$ & $3.6 \pm 0.6$ & .040 \\
External diameter (mm) & $47.4 \pm 5.1$ & $46.0 \pm 6.6$ & .527 \\
Wall thickness (mm) & $12.1 \pm 8.7$ & $0.6 \pm 4.7$ & .758 \\
Hospital death & $1(11 \%)$ & $1(4 \%)$ & .477 \\
\hline & & & \\
\hline
\end{tabular}

\section{Results}

Nine of 33 patients (27\%) underwent surgery on an emergency basis within 24 hours after admission and were included in the early surgery group. Five of 9 patients had been in shock on admission because of cardiac tamponade $(n=3)$ or aortic rupture $(n=2)$. Four patients had deterioration in condition after admission because of cardiac tamponade. Total arch replacement was performed in 3 patients, 2 with aortic rupture and 1 with cardiac tamponade associated with distal aortic arch aneurysm. Replacement of the ascending aorta was performed in 6 patients with cardiac tamponade. One of the 7 patients with cardiac tamponade required open drainage in the intensive care unit before surgery.

Twenty-four of 33 patients (73\%) were initially treated medically and included in the medical treatment group. Three patients had a history of type B IMH. Eleven patients in this group had high blood pressure $(>160 \mathrm{~mm} \mathrm{Hg})$ on admission. To maintain blood pressure at an appropriate level, 3 of these 11 patients required mechanical ventilation to put them under sedation.

Eight of 24 patients in the medical treatment group had conversion of their treatment to surgery during the follow-up period. Five of these 8 patients underwent surgery during the same hospitalization. One patient reported recurrent severe back pain 3 weeks after admission and had impending rupture diagnosed. Total arch replacement was done in this patient on an emergency basis. In 4 cases, IMH progressed to overt type A aortic dissection, which was discovered by serial CT scan performed 4 weeks after admission in the absence of any symptoms. Elective replacement of the ascending aorta was done in 3 cases and elective total arch replacement with aortic valve replacement was done in 1 case. In 2 patients, although IMH had disappeared at the time of discharge, overt type A aortic dissection was detected by serial CT scan performed 3 months after discharge. One patient underwent replacement of the ascending aorta, and the other underwent simultaneous ascending and descending aorta replacement because of association with progressive descending aortic dilatation. Another patient underwent total arch replacement 4 years after the initial episode of IMH with progressive aortic arch dilatation. The interval between the onset of IMH and conversion to surgery ranged from 24 days to 46 months.

The remaining 16 of 24 patients in the medical treatment group were treated only medically. The IMH of these 16 patients had completely regressed by 1 year after discharge, as confirmed by serial CT scans.

Table 1 presents comparisons of clinical characteristics and CT findings between the early surgery group and medical treatment group. The medical treatment group was younger than the early surgery group $(64.2 \pm 7.0$ years vs $71.7 \pm 8.5$ years, $P=.0319$ ). There were no statistically significant differences between the groups in terms of sex, hypertension, diabetes mellitus, hyperlipidemia, association of cerebrovascular disease, cardiac disease, lung disease, liver disease, renal dysfunction, abdominal aortic aneurysm, and history of type B IMH.

The extent of IMH was significantly greater in the medical treatment group than in the early surgery group (3.6 \pm 0.6 vs $3.0 \pm 0.9, P=.0395)$. The maximum diameter and wall thickness of the ascending aorta were not significantly different between two groups.

One patient in the early surgery group died after the operation. Although there were no IMH-related deaths in the medical treatment group, 1 patient died of sepsis resulting from an associated complication, liver abscess. In-hospital mortality rates in the early surgery group and the medical treatment group were $11 \%$ and $4 \%$, respectively $(P=.4773)$.

One patient in the early surgery group died of cerebral bleeding 59 months after surgery. Three patients in the medical treatment group died during the follow-up period: the first died of chronic renal failure 7 months later, the second died suddenly 18 months later and no autopsy was 


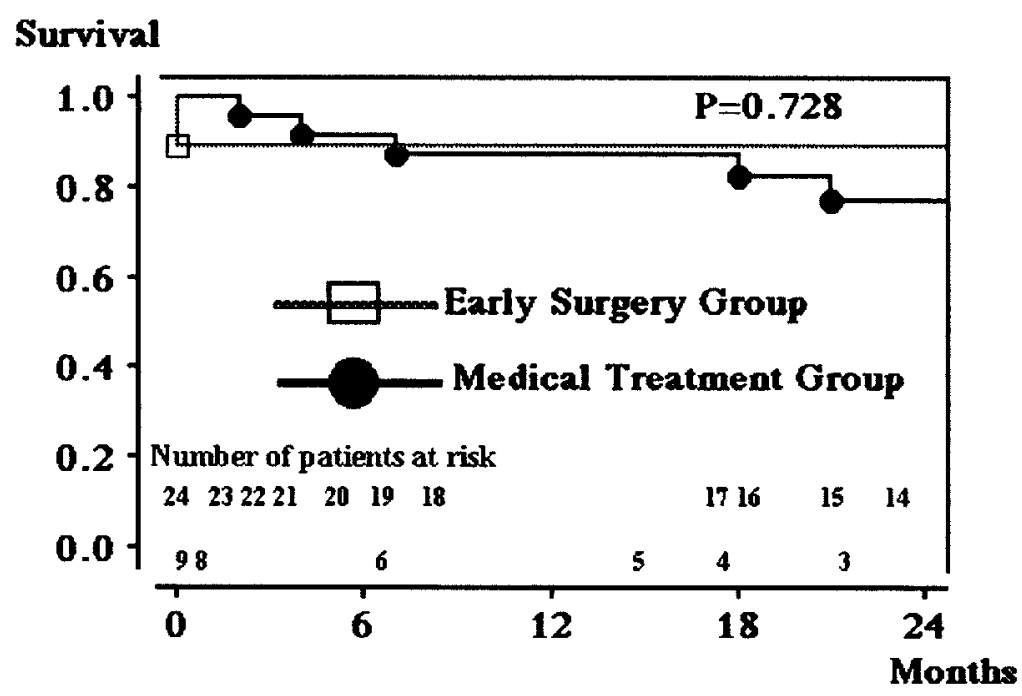

Figure 2. Cumulative survival curve by Kaplan-Meier method.

performed, and the third died of lung cancer 21 months later. The overall cumulative 1- and 2-year survivals were $89 \%$ and $89 \%$ in the early surgery group and $91 \%$ and $81 \%$ in the medical treatment group, respectively $(P=.728$; Figure 2).

Table 2 shows the results of univariate analysis of the clinical and CT image variables. Only the wall thickness in the CT images obtained 2 weeks after admission was found to be significantly correlated with conversion to surgery, and it was the only predictor of conversion of medical treatment to surgery throughout the entire follow-up period $(P=.038)$. We calculated an optimal cutoff value of the wall thickness at 2 weeks to predict progression to type A aortic dissection within 4 weeks and found $12 \mathrm{~mm}$ to be an optimal cutoff value, resulting in positive and negative predictive values of $80 \%$ and $100 \%$, respectively.

\section{Discussion}

IMH, the clinical presentation of which mimics that of acute aortic dissection, is considered a unique pathologic entity among aortic diseases. The process may arise from spontaneous rupture of the vasa vasorum ${ }^{12,13}$ or rupture of an atherosclerotic plaque. ${ }^{5,14}$ The prevalence of IMH is reported to be from $5 \%$ to $23 \%{ }^{4,15-17}$ of patients with acute aortic syndromes.

\section{Diagnostic Criteria and Confirmation}

The diagnosis of IMH is based on the evidence of wall thickness without intimal flap or disruption, by excluding aortic dissection and penetrating atherosclerotic ulcer. Furthermore, wall thickness with atherosclerosis and aortic dissection with thrombosed false lumen should be excluded.

Because the characteristic CT findings of IMH described by Yamada and coworkers ${ }^{12,18}$ have received wide recognition, we introduced them as one of our diagnostic criteria. On the basis of Yamada and coworkers' description ${ }^{12,18}$ alone, however, it is difficult to differentiate IMH from wall thickness with atherosclerosis or aortic dissection with thrombosed false lumen. Our second diagnostic criterion included having symptoms similar to those of aortic dissection and a time limit of 24 hours from the onset of symptoms to the initial CT examination. Patients with wall thickness with atherosclerosis usually have no symptoms. Furthermore, in patients with aortic dissection with thrombosed false lumen, the false channel may be opacified on contrast CT scan unless the thrombosing process occurred very rapidly (within 24 hours). Thus we considered that these criteria could exclude wall thickness with atherosclerosis and aortic dissection with thrombosed false lumen in all cases but those with very rapid thrombosis.

To obtain an accurate diagnosis of IMH, however, the diagnosis must be confirmed by further examinations. $\mathrm{Pa}-$ tients in the early surgery group had confirmation through operative findings. Patients in the medical treatment group had confirmation through serial CT findings, morphologic changes in aortic wall thickness that differed from wall thickness with atherosclerosis, and combined use of magnetic resonance imaging and digital subtraction angiography negative for aortic dissection and penetrating atheromatous ulcers ("giant ulcers"), as described in previous reports. ${ }^{4}$

Even though various invasive and noninvasive imaging techniques, such as $\mathrm{CT}^{5,9,12}$ scan, magnetic resonance imaging ${ }^{18-20}$, transesophageal echocardiography, ${ }^{8,21}$ aortography with digital subtraction angiography, ${ }^{22}$ and intravascular ultrasonographic imaging, ${ }^{11,23}$ have been used to 
TABLE 2. Results of univariate and multivariate analyses

\begin{tabular}{|c|c|c|c|c|c|}
\hline \multirow[b]{2}{*}{ Variable } & \multicolumn{2}{|c|}{ Univariate } & \multicolumn{3}{|c|}{ Multivariate } \\
\hline & $\chi^{2}$ & $P$ value & Hazard ratio & 95\% Confidence interval & $P$ value \\
\hline Age & 2.718 & .099 & & & \\
\hline Sex & 1.05 & .204 & & & \\
\hline Hypertension & 0.851 & .356 & & & \\
\hline \multicolumn{6}{|c|}{ CT examinations on admission } \\
\hline Extent of IMH & 0.102 & .749 & & & \\
\hline External diameter & 2.914 & .088 & & & \\
\hline Wall thickness & 0.023 & .879 & & & \\
\hline \multicolumn{6}{|c|}{ CT examinations 2 weeks later } \\
\hline External diameter & 1.678 & .195 & & & \\
\hline Wall thickness & 4.325 & .038 & 1.127 & $1.007-1.262$ & .038 \\
\hline
\end{tabular}

diagnose IMH, we used CT scanning for the initial diagnosis. CT is a useful modality not only for detecting aortic wall abnormalities and the location of aortic abnormalities but also for evaluating the presence of pericardial and pleural effusion or rupture into the mediastinum. These findings provided important information on whether to choose surgical or medical treatment. Furthermore, a high-speed fourrow detector CT system (Aquilion; Toshiba Corporation Medical Systems Company, Tokyo, Japan) is now available that can scan the entire aorta (from ascending to abdominal aorta) within half a minute. In consideration of the therapeutic approach, CT scanning is the first choice of diagnostic technique, especially in emergency situations.

\section{Management of Type A IMH}

According to previous reports, ${ }^{3-7,10,17,19,21}$ patients with type B IMH can be treated the same as those with type B aortic dissection. ${ }^{24}$ However, the optimal mode of management of patients with type A IMH, for whom previous clinical data are limited, remains controversial.

Some reports have noted that medical treatment alone leads to a poor prognosis for patients with type A IMH.3-7 Yamada and coworkers ${ }^{18}$ reported 6 cases of type A IMH. All 6 patients were treated medically, and 2 of them died within 1 month. Neiaber and colleagues ${ }^{4}$ reported that in their series of 12 patients with type A IMH, the 30-day mortality was $80 \%$ with medical treatment, versus $0 \%$ with early surgical repair $(P<.01)$, and they concluded that early surgical repair should be considered for all patients with type A IMH. Similar results were described by Harris and coworkers. ${ }^{17}$ Furthermore, Maraj and associates, ${ }^{7}$ in their meta-analysis of 143 reported cases of IMH, found that patients with type A IMH who underwent surgery had a significantly better prognosis than did those who received medical treatment (14\% vs 36\% mortality, respectively, $P<.02$ ). These reports noted the risk of progression of type A IMH to overt dissection or aortic rupture and the limitation of medical treatment alone in patients with type A IMH in their explanation of why early surgical repair is recommended.

On the other hand, considering the pathophysiologic process in IMH, absence of flow and pressure communication with the aortic lumen may produce good results. ${ }^{8-11}$ Kaji and associates ${ }^{9}$ reported that in-hospital mortality was $5 \%$ in 22 cases of type A IMH, even though all of patients were treated medically. Further, IMH of the ascending aorta regressed in 12 patients (55\%) and completely disappeared in 6 of these 12 patients. Taking into account these findings, the clinical course of type A IMH includes a whole spectrum of prognoses. In some cases immediate surgical repair may be indispensable, whereas in other cases hematoma within the aortic wall occasionally may be completely absorbed within a day or two. ${ }^{10,25}$

According to Movsowitz and colleagues' proposal for management of patients with penetrating atherosclerotic ulcer, we carried out early surgery only for patients with cardiac tamponade, impending rupture, or rupture. As a result, in-hospital mortality in the medical treatment group was $4 \%$, and 1 - and 2-year survivals were $92 \%$ and $81 \%$, respectively. These rates were not significantly different from those in the early surgery group $(11 \%, 89 \%$, and $89 \%$, respectively). We therefore considered only patients with type A IMH with cardiac tamponade, impending rupture, or rupture to be candidates for early surgery.

However, it is well known that type A IMH progresses to overt type A dissection with cardiac tamponade or aortic rupture in some medically treated patients, and such progressions carry a poor prognosis. Previous studies have shown that these progressions almost all occur within 7 days from the onset of IMH. ${ }^{4}$ This period could be considered an acute phase, like that of acute aortic dissection. ${ }^{24}$ The aortic wall is extremely fragile, and consequently type A IMH could easily progress to type A dissection with tamponade or aortic rupture. We consider that resting in bed with intensive antihypertensive therapy during this period could help to prevent such serious events. In our study, no med- 
ically treated patients with type A IMH had progression to overt type A dissection or aortic rupture within 2 weeks after the onset of IMH.

Although in 8 patients in the medical treatment group IMH progressed to overt type A dissection, impending rupture, or aortic dilatation during the follow-up period, all of these patients received opportune surgery. In 7 of 8 cases these progressions occurred within 3 months from the onset of IMH. By not missing the opportunity to convert from medical to surgical management, good long-term results were obtained. For that purpose, close follow-up for at least 3 months with serial CT scans or other imaging modalities is needed to evaluate the morphologic changes of IMH in patients undergoing medical treatment.

Finally, it is important to clarify the predictive factors of progression of IMH and conversion from medical treatment to surgery. Kaji and associates ${ }^{9}$ reported on 23 patients with type A IMH and concluded that the only significant predictor of progression of IMH (although their definition of progression was increasing maximum aortic wall thickness in follow-up CT images or death from rupture) was maximum aortic diameter in initial CT images. They noted that the optimal cutoff value was $50 \mathrm{~mm}$. Nishigami and colleagues ${ }^{27}$ reported on 44 patients with type A or type B IMH and mentioned that when overt aortic dissection occurred in patients with IMH, in all cases IMH was still demonstrated for longer than 1 month and the maximum aortic diameter was more than $45 \mathrm{~mm}$. Both studies pointed out that the maximum aortic diameter in initial CT images might have much to do with the progression of IMH. In our study, the only significant predictor of conversion to surgery was the wall thickness in CT images obtained 2 weeks after admission. Furthermore, we calculated an optimal cutoff value to predict progression to type A IMH within 4 weeks. A cutoff value of $12 \mathrm{~mm}$ in the wall thickness at 2 weeks was obtained, resulting in positive and negative predictive values of $80 \%$ and $100 \%$, respectively. This result suggests that medically treated patients with a wall thickness at 2 weeks less than $12 \mathrm{~mm}$ in diameter may not need to stay in hospital for longer than 2 weeks. Also, elective operation should be recommended for medically treated patients if wall thickness is more than $12 \mathrm{~mm}$ in diameter at 2 weeks. The number of our cases was as limited, however, as has been the case in previous reports, and further study may be needed.

There are a few limitations of this study. First, we compared the results of medical group with those of the surgical group and are lacking an appropriate control group. Actually, medically treated patients were less ill patients. We tried to discriminate the patients who should be treated surgically from the patients who could be treated medically. The next step would be to compare treatment strategies between groups with similar clinical statuses. Second, a 4-week hospital stay is surely long. We found that wall thickness at 2 weeks was the predictor of conversion to surgery, and an optimal cutoff value of $12 \mathrm{~mm}$ was determined. As a result, hospital stay could be shortened.

\section{Conclusion}

Early surgical treatment is required for patients with type A IMH combined with cardiac tamponade, impending rupture, or rupture. Patients without tamponade, impending rupture, or rupture may be candidates for medical treatment. However, serial imaging modalities, such as CT scan, magnetic resonance imaging, and transesophageal echocardiography, should be used, because in about $20 \%$ of cases IMH progresses to overt type A dissection or progressive aortic dilatation, and surgical repair is needed in such cases.

We thank Dr Shoko Komatsu for valuable help with statistical analysis.

\section{References}

1. Krukenberg E. Beiträge zur Frage des Aneurysma dissecans. Beitr Pathol Anat Allg Pathol. 1920;67:329-51.

2. Coady MA, Rizzo JA, Elefteriades JA. Pathologic variants of thoracic aortic dissections: penetrating atherosclerotic ulcers and intramural hematomas. Cardiol Clin. 1999;17:637-57.

3. Robbins RC, McManus RP, Mitchell RS, Latter DR, Moon MR, Olinger GN, et al. Management of patients with intramural hematoma of the thoracic aorta. Circulation. 1993;88(5 Pt 2):II1-10.

4. Nienaber CA, von Kodolitsch K, Petersen B, Loose R, Helmchen U, Haverich A, et al. Intramural hemorrhage of the thoracic aorta: diagnostic and therapeutic implications. Circulation. 1995;92:1465-72.

5. Muluk SC, Kaufman JA, Torchiana DF, Gertler JP, Cambria RP. Diagnosis and treatment of thoracic aortic intramural hematoma. Vasc Surg. 1996;24:1022-9.

6. von Kodolitsch Y, Nienaber CA. [Intramural hemorrhage of the thoracic aorta: diagnosis, therapy and prognosis of 209 in vivo diagnosed cases.] Z Kardiol. 1998;87:797-807.

7. Maraj R, Rerkpattanapipat P, Jacobs LE, Makornwattana P, Kotler MN. Meta-analysis of 143 reported cases of aortic intramural hematoma. Am J Cardiol. 2000;86:664-8.

8. Kang DH, Song JK, Song MG, Lee IS, Song H, Lee JW, et al. Clinical and echocardiographic outcomes of aortic intramural hemorrhage compared with acute aortic dissection. Am J Cardiol. 1998;8:202-6.

9. Kaji S, Nishigami K, Akasaka T, Hozumi T, Takagi T, Kawamoto T, et al. Prediction of progression or regression of type A aortic intramural hematoma by computed tomography. Circulation. 1999;100(19 Suppl):II281-6.

10. Vilacosta I, San Roman JA, Ferreiros J, Aragoncillo P, Mendez R, Castillo JA, et al. Natural history and serial morphology of aortic intramural hematoma: a novel variant of aortic dissection. Am Heart J. 1997;134:495-507.

11. Song JK, Kang DH, Lim TH, Song MG, Kim JJ, Park SW, et al. Different remodeling of descending thoracic aorta after acute event in aortic intramural hemorrhage versus aortic dissection. Am J Cardiol. 1999;83:937-41.

12. Yamada T, Takamiya M, Naito H, Kozuka T, Nakajima N. Diagnosis of aortic dissection without intimal rupture by x-ray computed tomography. Nippon Acta Radiol. 1985;45:699-710.

13. Gore I. Pathogenesis of dissecting aneurysm of the aorta. Arch Pathol Lab Med. 1952;53:142-53.

14. Murray CA, Edwards JE. Spontaneous laceration of the ascending aorta. Circulation. 1973;47:848-58.

15. Hirst AE, Johns VJ, Kime SW. Dissecting aneurysm of the aorta: a review of 505 cases. Medicine. 1958;37:217-79. 
16. Wilson SK, Hutchins GM. Aortic dissecting aneurysms: causative factors in 204 subjects. Arch Pathol Lab Med. 1982;106:175-80.

17. Harris KM, Braverman AC, Gutierrez FR, Barzilai B, Davila-Roman VG. Transesophageal echocardiographic and clinical features of aortic intramural hematoma. J Thorac Cardiovasc Surg. 1997;114:619-26.

18. Yamada T, Tada S, Harada J. Aortic dissection without intimal rupture: diagnosis with MR imaging and CT. Radiology. 1988;168:34752.

19. Murray JG, Manisali M, Flamm SD, VanDyke CW, Lieber ML, Lytle $\mathrm{BW}$, et al. Intramural hematoma of the thoracic aorta: MR image findings and their prognostic implications. Radiology. 1997;204:349-55.

20. Wolff KA, Herold CJ, Tempany CM, Parravano JG, Zerhouni EA. Aortic dissection: atypical pattern seen at MR imaging. Radiology. 1991;181:489-95.

21. Mohr-Kahaly S, Erbel R, Kearney P, Puth M, Meyer J. Aortic intramural hemorrhage visualized by transesophageal echocardiography: findings and prognostic implications. J Am Coll Cardiol. 1994;23:65864.
22. Bansal RC, Chandrasekaran K, Ayala K, Smith DC. Frequency and expansion of false negative diagnosis of aortic dissection by aortography and transesophageal echocardiography. J Am Coll Cardiol. 1995;25:1393-401.

23. Alfonso F, Goicolea J, Aragoncillo P, Hernandez R, Macaya C. Diagnosis of aortic intramural hematoma by intravascular ultrasound imaging. Am J Cardiol. 1995;76:735-8.

24. Miller DC, Stinson EB, Shumway NE. Realistic expectations of surgical treatment of aortic dissection: the Stanford experience. World J Surg. 1980;4:571-81.

25. Ohmi M, Tabayashi K, Moizumi Y, Komatsu T, Sekino Y, Goko C. Extremely rapid regression of aortic intramural hematoma. $J$ Thorac Cardiovasc Surg. 1999;118:968-9.

26. Movsowitz H, Lampert C, Jacobs LE, Kotler N. Penetraiting atherosclerotic aortic ulcers. Am J Cardiol. 1994;128:1210-7.

27. Nishigami K, Tsuchiya T, Shono H, Horibata Y, Honda T. disappearance of aortic intramural hematoma and its significance to the prognosis. Circulation. 2000;102 Suppl 3:III243-7.

\section{ON THE MOVE?}

Don't miss a single issue of the journal! To ensure prompt service when you change your address, please photocopy and complete the form below.

Please send your change of address notification at least six weeks before your move to ensure continued service. We regret we cannot guarantee replacement of issues missed due to late notification.

\section{JOURNAL TITLE:}

Fill in the title of the journal here.

\section{OLD ADDRESS:}

Affix the address label from a recent issue of the journal here.

\section{NEW ADDRESS:}

Clearly print your new address here.

Name

Address

City/State/ZIP

\section{COPY AND MAIL THIS FORM TO:}

Mosby

Subscription Customer Service

6277 Sea Harbor Dr

Orlando, FL 32887
OR FAX TO:

407-363-9661

N/ Mosby
OR PHONE:

800-654-2452

Outside the U.S., call

407-345-4000 